\title{
La calidad de una página web como herramienta de comunicación
}

\author{
David GRÁVALOS MACHO \\ Universidad Europea de Madrid \\ david.gravalos@uem.es
}

Recibido: 08/10/2012

Aceptado: 23/01/2013

\section{Resumen}

Planteando y excluyendo los modelos de negocio basados en internet, se hace interesante partir de una visión pragmática sobre la necesidad de crear una página web como herramienta de comunicación persuasiva. A partir de un hilo argumental lógico se marcan las pautas que permitirán establecer los indicadores de calidad, los cuales, a su vez, comprenden aspectos a nivel estratégico y táctico en su gestión. Valorar y detectar dichos indicadores que hacen posible mejorar un site o comparar varios es objeto de trabajo del comunicador. La reflexión sobre qué se necesita de una página web y para qué, son sus objetivos principales. Dicha reflexión deja abiertas las líneas de actuación necesarias para su correcta utilización y posterior análisis, las cuales serán desarrolladas por separado.

Palabras clave: Comunicación persuasiva, SEO, SEM, Posicionamiento web, Accesibilidad, Usabilidad, Arquitectura de la Información, Diseño web.

\section{The Quality of a Website as a Communication Tool}

\begin{abstract}
In order to create and manage a Website as a persuasive communication tool, the most important and useful method begins by identifying and excluding all business models based on the Web. Using logical arguments, all of the quality indicators dealing with strategic and tactical aspects in terms of managing a Website will be given. The communicators' main task is to detect and assess such indicators in order to improve a particular Website or compare various sites. The main objective of the communicators should be to think about those aspects necessary for a Website and why they are needed. This reflection leaves open necessary lines of action for their proper use and analysis, which will be developed separately.

Keywords: Persuasive communication, SEO, SEM, Web Positioning, Accessibility, Usability, Information Architecture, Web Design.

\section{Referencia normalizada}

GRÁVALOS MACHO, David (2013): "La calidad de una página web como herramienta de comunicación”. Estudios sobre el Mensaje Periodístico. Vol. 19, Núm. especial marzo, págs.: 253-261. Madrid, Servicio de Publicaciones de la Universidad Complutense.
\end{abstract}

Sumario: 1. Introducción: el concepto de comunicación. 2. Metodología. 3. Desarrollo; 3.1. Las Tecnologías de la Comunicación y la Información; 3.2. Los criterios de calidad de un entorno web; 3.2.1. Posicionamiento; 3.2.2. Accesibilidad; 3.2.3. Usabilidad; 3.2.4. Diseño visual; 3.2.5. Arquitectura de la información. 4. Conclusiones. 5. Referencias bibliográficas.

\section{Introducción: el concepto de comunicación}

El estudio de la comunicación constituye una disciplina humanística cuya importancia, cada vez mayor, se deriva del progreso tecnológico y de los cambios socioculturales que éste promueve. Tal estudio armoniza una serie de conocimientos teóricos con los resultados obtenidos empíricamente en campos científicos especializados y a veces altamente distantes.

Este carácter multidisciplinar y el nutrido y variado número de estudiosos y profesionales relacionados con el tema han dado lugar a la existencia de diversas defini- 
ciones del concepto comunicación y, por tanto, a un estimulante debate en cuanto a las dimensiones del mismo.

La extensión del concepto de comunicación ha supuesto una situación que lo ha llevado a la inexistencia de una definición única en el seno de un paradigma científico concreto.

Fauconnier, en un estudio de las diferentes acepciones de comunicación (Fauconnier, 1975: 9), registró un número cercano a cincuenta definiciones llegando a la conclusión de que las diferencias entre las mismas estaban motivadas por las disciplinas de las que procedían. En un intento de poder realizar una aproximación al concepto que contenga todo el extenso espectro de disciplinas y modalidades conectadas al mismo, se destacarán algunas de las definiciones más relevantes del término.

Martín Serrano afirma que la comunicación es "la capacidad que poseen los seres vivos de cambiar información con otros seres vivos. A todo ser vivo capaz de cambiar información, le conviene el título de "actor de la comunicación".[...] Se infiere que la aptitud para comunicar la posee el hombre y antes que él ya la habían adquirido muchas especies animales, incluso aquellas que están evolutivamente muy alejadas de la especie humana" (Martín Serrano, 1981: 11).

También hay que considerar el planteamiento propuesto por Sanabria cuando entiende que el hombre comunica o puede comunicarse con una máquina, con un animal o con otro hombre, pero que la comunicación humana no se acaba ahí, sino que implica a las partes, hace que entre los sujetos de la actividad comunicativa se establezca una relación mutua, la cual no se da ni respecto del animal ni respecto de la máquina. "¿Qué tiene de particular esa comunicación que la diferencia de otras, si se admite que hay otras? La comunicación humana, además de producirse sólo entre hombres y precisamente porque se produce entre ellos, es una comunicación simbólica y relacionante" (Sanabria, 1975: 42).

Moles, a partir del modelo cibernético va más allá, llegando a sostener que el hombre se distingue de otros sistemas biológicos por la extensión de su facultad de comunicación y ésta, en su sentido más amplio, la entiende como: "la acción de hacer participar a un individuo - o un organismo - situado en una época, en un punto R dado, en las experiencias y estímulos del entorno de otro individuo - de otro sistema - situado en otra época, en otro lugar E, utilizando los elementos de conocimiento que tienen en común" (Moles, 1975: 119).

Desde una posición más moderada, la sociología también hace objeto de su estudio al concepto de comunicación y, así, autores como Gerbner la definen como "la interacción social mediante mensajes, entendiéndose por éstos los eventos o aconteceres formalmente codificados, simbólicos o representacionales, que cuentan con una significación en una cultura dada, y que se producen con el propósito de evocar significación" (Arceo, 1988a: 10).

De las anteriores definiciones se puede desprender, por tanto, que no existe una opinión general y unánime del concepto, y así es considerado por Sanabria cuando advierte que apenas existen definiciones y las que hay no son de aceptación general; no ya porque se discrepe sobre su exactitud o su precisión, lo que sigue ocurriendo respecto de conceptos tratados desde muy antiguo, sino porque tales definiciones care- 
cen de extensión y se limitan intencionadamente a aspectos parciales que interesan a su autor o al campo de estudio en que se mueven (Arceo, 1988b: 41).

Dejando a un lado otras áreas de conocimiento y sus diversos conceptos de comunicación, consideramos que la comunicación humana es la actividad cognitiva de interacción que se produce al menos entre dos personas, emisor y receptor, con capacidad de generar mensajes, con o sin empleo de medios técnicos, en los cuales subyace un proceso de representación común entre ambos.

\section{Metodología}

La metodología que se llevará a cabo en este artículo y como procedimiento de investigación y desarrollo parte de estructurar las páginas web en ítems que nos acerquen a entender el proceso comunicativo y para ello se despliega desde el método inductivo, procediendo de la singularidad de sus contenidos y sus textos hasta la generalización de la eficacia comunicativa que ha de llevar. Así pues se entenderá y analizará el razonamiento desde lo particular a lo más general y desde el mismo conocimiento prematuro de la comunicación persuasiva.

\section{Desarrollo}

\subsection{Las Tecnologías de la Comunicación y la Información}

Internet constituye una revolución del conocimiento humano, centrada en su capacidad de comunicarse y transformarse. Se ha logrado construir en relación con las nuevas tecnologías de la información y la comunicación, a todos los medios de comunicación tradicionales (prensa, radio y televisión). A partir de este concepto, los medios de comunicación constituyen las diferentes formas de estas tecnologías, puesto que representan una aplicación del conocimiento humano, destinada a mejorar sus procesos comunicativos.

Los medios de comunicación se constituyen, en consecuencia, como una prolongación de las tecnologías de información que responden a nuevas necesidades de comunicación humana, suponiendo nuevas formas de transmitir y recibir información, que permiten nuevos procesos de producción.

Los aportes que Internet ha hecho a los medios como instrumentos técnicos de comunicación son diversos. Entre otros se pueden citar, la mejora y optimización de sus singulares procesos de producción, la forma de transmisión de sus mensajes y el modo de relacionarse con sus públicos, sin olvidar, como es obvio, los aspectos organizativos y gerenciales como entes colectivos que son. Este fenómeno, llamado Internet, tiene una característica que lo hace especialmente singular, pues se trata de una herramienta que posibilita a las personas poder acceder, de forma casi instantánea, a cantidades infinitas de información, con un costo relativamente bajo, sobre cualquier tipo de materia y procedente de cualquier rincón del planeta.

Este es el argumento con el que las empresas, instituciones y gobiernos se dejaron seducir por las infinitas posibilidades que, teóricamente, ofrecían las emergentes tecnologías, agrupadas todas en un concepto conocido como TIC (Tecnologías de la Información y la Comunicación), entre las cuales, Internet es la piedra angular.

Toda esta nueva forma de hacer negocios es conocida como, e-business, negocios electrónicos, que no son una simple forma de hacer negocio o un canal de venta y re- 
lación entre la organización y el cliente, sino que va más allá, se trata de una iniciativa de negocio que transformará sus relaciones, es una nueva forma de gestionar la creación de valor a la empresa, su eficiencia, innovación y velocidad.

Para materializar el uso de las TIC en el tejido empresarial, es esencial distinguir entre la implementación de un e-commerce y un e-business, ya que éste, ha sido uno de los factores que las empresas, de forma mayoritaria, no han sabido diferenciar en España. El negocio electrónico es utilizar la red para mejorar los procesos y tener, por tanto, un mejor desempeño, lo cual no implica vender algo por Internet, como es el caso del e-commerce. De esta manera, entenderemos el e-commerce como un proceso del negocio, "como la aplicación de la tecnología hacia la automatización de las transacciones de los negocios y el flujo del trabajo" (Siebel, 2001: 52).

Rappa (2011: http://www.digitalenterprise.org), desde una perspectiva básica, determina que un modelo de negocio es el método mediante el cual una empresa realiza negocios para generar beneficios.

El e-business es una nueva manera de gestionar las eficiencias, la velocidad, la innovación y la creación nuevo valor en una empresa.

Además de abarcar el e-commerce, el e-business incluye tanto las aplicaciones front-office (pública) como las back office (administrativa) que forman el núcleo de los negocios modernos. Así, el e-business no es, tan sólo una transacción de e-commerce o realizar una compra/venta por Internet, sino que es también la estrategia global de redefinir antiguos modelos de negocio, con la ayuda de la tecnología para maximizar el valor del cliente y, por tanto, las ganancias. El e-business incorpora el uso estratégico de las TIC para interactuar con clientes, proyectos, y socios a través de los canales de distribución adecuados para que le permitan una comunicación múltiple.

Es por ello, que surge la necesidad de estudiar y poner especial interés en los Key Facts (aspectos clave) que nos indiquen cuándo es necesario actuar. La realidad es que toda empresa, independientemente de su tamaño, industria o ámbito geográfico de operación, debe encontrar cómo adaptar su manera de hacer negocios y reconocer que Internet es la fuerza principal que conecta tanto a las empresas como a sus públicos.

Internet implica cambios sociales y económicos. Este nuevo canal permite una comunicación, imposible anteriormente, con recursos mínimos. Cualquier individuo o sociedad puede hacer llegar su mensaje al resto del mundo. Pero sólo esto no es comunicar de manera efectiva, para ello debe establecerse un canal de retroalimentación.

\subsection{Los criterios de calidad de un entorno web}

Después de haber establecido en las anteriores páginas el contexto de un uso apropiado de Internet como herramienta de comunicación, a través de los términos y definiciones dadas, es el momento de llegar a fundamentar los objetivos que hilan su instrumentalización. Para hacer un uso y explotación eficientes de Internet como herramienta de comunicación persuasiva, deben tomarse decisiones tanto a nivel estratégico como táctico, items que se convierten en el objetivo del presente trabajo, siendo éstos el posicionamiento, la accesibilidad, la usabilidad, el diseño y la arquitectura de la información. 
Los criterios desarrollados a continuación, deben entenderse en su conjunto ya que en multitud de ocasiones se influyen mutuamente. Sin embargo, para permitir una mejor comprensión de los mismos, se pueden detallar por separado.

\subsubsection{Posicionamiento}

Entendemos que el posicionamiento o SEP (Search Engine Positioning) se resume en la capacidad por parte del emisor para aparecer entre los resultados que un buscador ofrece cuando un internauta realiza una búsqueda determinada. Persiguiendo tal fin, el emisor ha de definir ciertas palabras clave con las que será encontrado a través de los buscadores. Las palabras clave, o keywords, deben ser entendidas como aquéllas palabras que definen al objeto de comunicación del emisor pero que, además, coincidan con un interés contrastado por parte del receptor. El emisor precisa conocer previamente, aquéllos temas de interés del público al que se dirige y que, por ende introduce de manera proactiva en los motores de búsqueda.

A partir de el desarrollo de dichas palabras clave, realizará acciones para posicionarse en los resultados que el buscador ofrece y conseguir ser visitado por su target. Estas acciones se dividen en dos grandes grupos conocidos como $S E O$ (Search Engine Optimization) y $S E M$ (Search Engine Marketing) cuyo resultado define el propio posicionamiento o $S E P$.

Search Engine Optimization -SEO-: conjunto de técnicas orientadas a facilitar la navegación del buscador por nuestra página - pull-. Éstas son, transparencia en el código fuente - $\mathrm{html}$ - y construcción estructural del site (nombres de archivos y carpetas del Website, tags o etiquetas, descripciones alternativas, etc.).

Search Engine Marketing -SEM-: conjunto de técnicas orientadas a llamar la atención del buscador - push-. Estas son, alta y registro en los buscadores más utilizados $\mathrm{y}$ otras acciones fuera del site a fin de dotar de popularidad al sitio web.

Search Engine Positioning: los motores de búsqueda establecen, a través de un algoritmo, el criterio por el cual ofrecerán como primer resultado a un site, antes que a otro. Google, por ejemplo, lo llama PageRank, y consiste en una valoración que responde a la suma de cuán transparente es la página a ojos de una máquina $-S E O$ - y al índice de popularidad frente al resto -SEP-. Este algoritmo es cambiante en algunos aspectos y también atiende a un criterio geográfico sobre el origen de la búsqueda. De esta manera, el resultado a nivel local sobre un establecimiento cercano dará prioridad a la misma zona geográfica en que se encuentre el usuario que realiza la búsqueda frente a otra zona diferente. El criterio del posicionamiento es determinante, ya que es el origen de cualquier tipo de comunicación posible que una organización pueda tener con sus públicos a través de la red.

\subsubsection{Accesibilidad}

La accesibilidad (Fuertes Castro, 2007: 135-154) es la puesta en práctica de un conjunto de técnicas orientadas a una correcta navegación del internauta. La referencia comúnmente aceptada a nivel mundial cuando se habla de accesibilidad web es la proporcionada por el Consorcio de la Web (w3c, 2006), abreviado como $w 3 c$, del inglés World Wide Web Consortium, a través de la Iniciativa para la Accesibilidad a la 
Web (WAI, 2006), abreviada como WAI, del inglés Web Accessibility Initiative. Según datos del INE - Instituto Nacional de Estadística -, España cuenta con casi cuatro millones de personas discapacitadas (INE, 2012). Esto supone casi un 10\% de la población.

La accesibilidad web se encuentra regulada, desde el año 1999, a través de la guía WCAG (Web Content Accessibility Guidelines) y, en España cuenta con el respaldo de la norma técnica española de AENOR UNE 139803:2004 (AENOR, 2004). WCAG plantea un total de 65 puntos de control, organizados en catorce14 pautas de alto nivel. Dos grandes principios dirigen estas catorce pautas. El primero transformación elegante, que indica que las páginas web deben diseñarse para que se adapten de forma adecuada a las distintas posibilidades de navegación que pueden presentarse, como, tipos de dispositivos, tipos de navegadores, características de visualización, etc. El primer principio agrupa a la mayoría de las pautas, desde la uno a la once. El segundo principio es que los contenidos deben ser fáciles de comprender y de navegar.

A pesar de la relativa facilidad que tiene lograr que la mayoría de elementos web sean accesibles (uso de $x \mathrm{html}$ y css), resulta paradójica la dificultad que existe para encontrar sitios web accesibles. Existen también ciertos mitos que merecen ser desmentidos, como justificar que una web no sea accesible debido al escaso número de discapacitados, o que el target de un espacio web no incluye a ningún discapacitado. Contra esto podemos argumentar que un segmento de un $10 \%$ del universo poblacional abarcable por una empresa, bien merece la confección de una estrategia de marketing para ofrecerles productos que satisfagan sus necesidades. Por otro lado, es conveniente la reflexión de que en los procesos de compra, existe la figura del prescriptor, el cual, aunque no sea un consumidor final, puede inclinar la balanza de decisiones por recomendación de dicho sujeto, y éste puede tener una discapacidad. Finalmente cabe subrayar que si un sitio es accesible para alguien con discapacidad, se infiere directamente que también lo es para el resto de la población, además de ciertas máquinas entre las que se encuentran los buscadores, lo cual incidirá (según técnicas de SEO) en un posicionamiento mejor.

\subsubsection{Usabilidad}

Relativo a la usabilidad y como elemento estrechamente relacionado al resto de criterios, cabe definirla como "el atributo de calidad que mide lo fáciles que son de usar las interfaces Web" (Nielsen, 2006: 18).

La Asociación Internacional para la Estandarización (ISO) la define como "capacidad de un software de ser comprendido, aprendido, usado y ser atractivo para el usuario, en condiciones específicas de uso (ISO, 2012). Y su aplicación práctica se recoge en el estándar ISO-9126. (ISO/IEC 9126-1, 2012). Entendiendo estas normas como el conjunto de técnicas orientadas a una correcta Interacción Persona-Ordenador $(I P O / H C I)$, podemos resumir sus aspectos más importantes, poniendo interés en los tres focos que son detallados a continuación. Facilidad de aprendizaje: la facilidad con la que nuevos usuarios pueden tener una interacción efectiva. Está relacionada con la predicibilidad, sintetización, familiaridad, la generalización de los conocimientos previos y la consistencia. flexibilidad: la variedad de posibilidades con las 
que el usuario y el sistema pueden intercambiar información. También abarca la posibilidad de diálogo, la multiplicidad de vías para realizar la tarea, similitud con tareas anteriores y la optimización entre el usuario y el sistema. La robustez es el nivel de apoyo al usuario que facilita el cumplimiento de sus objetivos. Está relacionada con la capacidad de observación del usuario, de recuperación de información y de ajuste de la tarea al usuario.

\subsubsection{Diseño visual}

Como cuarto objetivo planteado en este trabajo, cabe destacar el más tenido en cuenta por todos los desarrolladores y usuarios de Internet: el diseño. El proceso de diseño, más allá de la herramienta de diseño que se elija, debe responder a aspectos técnicos, creativos y estratégicos (Valdés-Miranda, 2005: 78). Este es un aspecto muy discutido y muy discutible que ofrece infinidad de autores que lo abordan, tanto desde una perspectiva científica como desde una perspectiva de generación de impacto. Dentro de dicho objetivo debemos plantear su aporte contextualizado en la investigación, para lo cual, lo tomaremos como un indicador de calidad. Ello nos obliga a estructurarlo de acuerdo a un análisis desde una triple perspectiva ya que comienza a involucrarse con el resto de objetivos. Éstos son, por tanto, su análisis desde una perspectiva icónica, cromática y lingüística para definir su adecuación al objetivo comunicacional. Siguiendo esto, el estudio analizará desde un punto de vista icónico su idoneidad de acuerdo a los criterios de usabilidad, desde un nivel cromático debemos analizar su integración con la identidad visual corporativa y su significación cromática de acuerdo a la cultura de los públicos objetivo y su análisis lingüístico bajo los parámetros establecidos en sus requerimientos de comunicación persuasiva.

\subsubsection{Arquitectura de la información}

Por último, el objetivo de la arquitectura de la información se establece como elemento integrador más estratégico de los planteados. Podemos definir como: "la disciplina y arte encargada del estudio, análisis, organización, disposición y estructuración de la información en espacios de información, y de la selección y presentación de los datos en los sistemas de información interactivos y no interactivos." (Pérez-Montoro, 2010: 333-338).

Se trata, sin duda de un esfuerzo empático, resumido en un conjunto de acciones orientadas a transmitir los mensajes corporativos y, al mismo tiempo, satisfacer las necesidades de información de los públicos de la organización en el menor número de clicks posible. No es recomendable superar tres clicks. (López Gil, 2010: 359-367).

Es una variable de carácter estratégico y debe ser integrado en los planes de comunicación persuasiva de la organización como un elemento de suma importancia, por su capacidad de interactuar con todos los segmentos de la empresa.

\section{Conclusiones}

Los objetivos que engloban la instrumentalización en el contexto de un uso apropiado de internet desde la perspectiva comunicacional, pasa por el posicionamiento, la accesibilidad, usabilidad, diseño y arquitectura de la información. 
El emisor, a través de una definición contrastada de palabras clave, tendrá que conocer los temas por los que el público muestre un interés proactivo en los motores de búsqueda.

La accesibilidad, siempre regulada, conlleva una transformación que fundamente las páginas web en su diseño y aceptación de las posibilidades existentes de navegación de acuerdo a los dispositivos existentes en el mercado.

La flexibilidad, robustez del sistema y facilidad de aprendizaje, hacen posible la optimización de interacción usuario-sistema en lo relativo a la usabilidad, estableciendo sinergias entre los distintos criterios existentes que buscan ofrecer un buen uso de los interfaces web.

Tanto el diseño visual como la arquitectura de la información son objetivos fiables que aportan una contextualización en la investigación científica como referente de calidad, atendiendo a su grado de iconicidad, cromatismo y lingüística para establecer una eficaz estrategia comunicacional.

\section{Referencias bibliográficas}

ARCEO VACAS, José Luis (1988): Fundamentos para la teoría y técnica de las relaciones públicas. 1 edn. Promociones y Publicaciones Universitarias, PPU, pp. $10-41$.

FAUCONNIER, Guillaume (1975): Mass Media and Society. Leuven University Press.

FUERTES CASTRO, Jose Luis y MARTÍNEZ NORMAND, Loïc (2007): Accesibilidad web. Revista De Traductología, (11). Disponible en http://dialnet unirioja .es.delfos.uem.es/servlet/articulo? codigo $=2306685$ \&orden $=180314 \&$ info $=\operatorname{link}[\mathrm{Fe}$ cha de consulta: 20 de agosto de 2012].

INSTITUTO NACIONAL DE ESTADÍSTICA (INE, 2012): Población con alguna discapacidad o limitación por edad o sexo. Disponible en: http://www.ine.es /jaxi/tabla.do?path=/t15/p418/a2008/hogares/p01/modulo1/10/\&file=01001.px\&ty pe $=$ pcaxis $\& L=0$. [Fecha de consulta: 2 de febrero de 2012].

ISO-INTERNATIONAL ORGANIZATION FOR STANDARDIZATION. Disponible en http://www.iso.org/iso/home.htm. [Fecha de consulta: 20 de agosto de 2012]

ISO/IEC 9126-1:2001 - Software engineering — Product quality - Part 1: Quality model. Disponible en http://www.iso.org/iso/iso_catalogue/catalogue_tc/catalogue_detail.htm?csnumber=22749. [Fecha de consulta: 20 de agosto de 2012].

LÓPEZ GIL, Juan Miguel et al (2010): “Análisis de la arquitectura de webs mediante tests de estrés de navegación, de usabilidad y eye tracking”. El Profesional De La Información, 19(4). Barcelona.

MARTÍN SERRANO, Manuel et al. (1981): Epistemología de la comunicación y análisis de la referencia. Madrid, Ed. Cuadernos de la Comunicación. Vol. VIII.

MOLES, Abraham y ZELTMANN, Claude (1975): La Comunicación y los mass media. Voz Comunicación. Bilbao, Ediciones Mensajero. 
NIELSEN, Jakob et al. (2006): Usabilidad. prioridad en el diseño web (1st ed.) ANAYA MULTIMEDIA, p. 18. [traducción, Eva Gallud Jurado].

PÉREZ-MONTORO GUTIÉRREZ, Mario (2010): “Arquitectura de la información en entornos web”. El Profesional De La Información, 19(4). Barcelona.

RAPPA, Michael: Business Models on the Web Managing the Digital Enterprise. Disponible en http://www.digitalenterprise.org. [Fecha de consulta: 2 de febrero de 2011].

SANABRIA MARTÍN, Francisco (1975): Estudios Sobre Comunicación. Madrid, Editora Nacional

SIEBEL, Thomas (2001): Principios del e-business: Cómo los líderes actuales del mercado aumentan los ingresos, la productividad y la satisfacción del cliente (1st ed.) Ediciones Juan Granica, p. 52.

VALDÉS-MIRANDA, Claudia y RODRÍGUEZ ÁLVAREZ, Enrique (2005). Creación y diseño web. ( $1^{\mathrm{a}}$ ed.) ANAYA MULTIMEDIA.

\section{David GRÁVALOS MACHO}

Universidad Europea de Madrid

Departamento de Publicidad y Contenidos

Profesor de la Facultad de Artes y Comunicación

david.gravalos@uem.es 